\title{
Multicenter Retrospective Analysis of Clinical Characteristics, Treatment Patterns, and Outcomes in Very Elderly Patients with Diffuse Large B-Cell Lymphoma: The Korean Cancer Study Group LY16-01
}

\author{
Jung Hye Choi, MD, PhD ${ }^{1}$ \\ Tae Min Kim, MD, PhD² \\ Hyo Jung Kim, MD, $P h D^{3}$ \\ Sung Ae Koh, MD, PhD ${ }^{4}$ \\ Yeung-Chul Mun, MD, PhD ${ }^{5}$ \\ Hye Jin Kang, MD, PhD ${ }^{6}$ \\ Yun Hwa Jung, $\mathrm{MD}^{7}$ \\ Hyeok Shim, MD \\ So Young Chong, MD, $\mathrm{PhD}^{9}$ \\ Der-Sheng Sun, MD, PhD'10 \\ Soonil Lee, MD, PhD ${ }^{11}$ \\ Byeong Bae Park, MD, PhD'12 \\ Jung Hye Kwon, MD, PhD'13 \\ Seung-Hyun Nam, MD ${ }^{14}$ \\ Jun Ho Yi, MD, PhD 15 \\ Young Jin Yuh, MD ${ }^{16}$ \\ Jong-Youl Jin, MD, PhD'17 \\ Jae Joon Han, MD, PhD ${ }^{18}$ \\ Seok-Hyun Kim, MD, PhD ${ }^{19}$
}

${ }^{*}$ A list author's affiliations appears at the end of the paper.

Correspondence: Jung Hye Choi, MD, PhD

Department of Internal Medicine,

Hanyang University Guri Hospital,

153 Gyeongchun-ro, Guri 11923, Korea

Tel: 82-31-560-2162

Fax: 82-31-553-7369

E-mail: jhcmd@hanyang.ac.kr

Received April 9, 2017

Accepted June 7, 2017

Published Online June 9, 2017

\section{Purpose}

The treatment strategy for elderly patients older than 80 years with diffuse large B-cell lymphoma (DLBCL) has not been established because of poor treatment tolerability and lack of data.

\section{Materials and Methods}

This multicenter retrospective study was conducted to investigate clinical characteristics, treatment patterns and outcomes of patients older than 80 years who were diagnosed with DLBCL at 19 institutions in Korea between 2005 and 2016.

\section{Results}

A total of 194 patients were identified (median age, 83.3 years). Of these, 114 patients had an age-adjusted International Prognostic Index (aalPI) score of 2-3 and 48 had a Charlson index score of 4 or more. R-CHOP was given in 124 cases, R-CVP in 13 cases, other chemotherapy in 17 cases, radiation alone in nine cases, and surgery alone in two cases. Twenty-nine patients did not undergo any treatment. The median number of chemotherapy cycles was three. Only 37 patients completed the planned treatment cycles. The overall response rate from 105 evaluable patients was $90.5 \%$ (complete response, 41.9\%). Twentynine patients died due to treatment-related toxicities (TRT). Thirteen patients died due to TRT after the first cycle. Median overall survival was 14.0 months. The main causes of death were disease progression (30.8\%) and TRT (27.1\%). In multivariate analysis, overall survival was affected by aalPI, hypoalbuminemia, elevated creatinine, and treatment.

\section{Conclusion}

Age itself should not be a contraindication to treatment. However, since elderly patients show higher rates of TRT due to infection, careful monitoring and dose modification of chemotherapeutic agents is needed.
Key words

Diffuse large B-cell lymphoma, Aged, Infection 


\section{Introduction}

There has been an increase in the number of elderly patients with malignant disease due to rapid growth of the elderly population. Despite the development of new drugs and improvements in supportive care, treatment and the cost of patient management are emerging as important medical issues in many countries. Diffuse large B-cell lymphoma (DLBCL), the most common type of non-Hodgkin's lymphoma, is a representative disease with increased incidence and poor prognosis in elderly patients. After introduction of rituximab (chimeric anti-CD20 monoclonal antibody), treatment outcome and prognosis of DLBCL has dramatically improved. R-CHOP (rituximab, cyclophosphamide, adriamycin, vincristine, and prednisone) is standard treatment for DLBCL $[1,2]$. However, most large randomized trials enrolled patients younger than 80 years. In addition, elderly patients do not tolerate treatment to the same extent as younger patients because of comorbid diseases and organ dysfunction. Therefore, treatment strategies for very elderly patients older than 80 years have not been established because of poor tolerability and lack of data.

Recently, a multicenter, single-arm phase 2 trial reported that rituximab combined with low-dose CHOP (R-miniCHOP) showed good results for both efficacy and safety in elderly patients older than 80 years with DLBCL [3]. Although this study was a phase 2 trial, the results suggested that very elderly patients could tolerate and benefit from aggressive combination chemotherapy with immunotherapy. However, no prospective data are available and only a few retrospective studies with a small number of patients over 70 years old have been conducted in Korea $[4,5]$. The aim of the current study was to retrospectively investigate clinical characteristics, treatment patterns and outcomes of patients older than 80 years diagnosed with DLBCL in Korea over a 10-year period. Based on our results, we planned to perform a prospective study to identify the optimal regimen for treating DLBCL elderly patients in Korea.

\section{Materials and Methods}

This study included patients who were older than 80 years at the time of initial DLBCL diagnosis from across 19 institutions in Korea between January 2005 and June 2016. A diagnosis of DLBCL was established via tissue biopsy according to the WHO classification. Clinical and laboratory data were retrospectively obtained from hospital records.

Disease and patient-specific characteristics included age,
Table 1. Patient characteristics

\begin{tabular}{|c|c|}
\hline Characteristic & No. $(\%)(n=194)$ \\
\hline \multicolumn{2}{|l|}{ Sex } \\
\hline Male & $93(47.9)$ \\
\hline Female & $101(52.1)$ \\
\hline Age, median (range, yr) & $83.3(80.1-95.7)$ \\
\hline \multicolumn{2}{|l|}{ Performance status (ECOG) } \\
\hline $0-1$ & $88(45.4)$ \\
\hline $2-4$ & $106(54.6)$ \\
\hline \multicolumn{2}{|l|}{ Ann Arbor stage $(n=193)$} \\
\hline I-II & $81(42.0)$ \\
\hline III-IV & $112(58.0)$ \\
\hline \multicolumn{2}{|l|}{ LDH level (n=192) } \\
\hline Normal & $73(38.0)$ \\
\hline Abnormal & $119(62.0)$ \\
\hline \multicolumn{2}{|l|}{ No. of extranodal sites $(n=193)$} \\
\hline$<2$ & $154(79.8)$ \\
\hline$\geq 2$ & $39(20.2)$ \\
\hline \multicolumn{2}{|l|}{ aaIPI (n=192) } \\
\hline 0 & $22(11.5)$ \\
\hline 1 & $56(29.2)$ \\
\hline 2 & $64(33.3)$ \\
\hline 3 & $50(26.0)$ \\
\hline B symptoms & $41 / 176(23.3)$ \\
\hline Bulky disease & $15 / 185(8.1)$ \\
\hline Elevated $\beta 2$-microglobulin & $64 / 89(71.9)$ \\
\hline Anemia (hemoglobin < 12 g/dL) & $118 / 194(60.8)$ \\
\hline Thrombocytopenia & $36 / 194(18.6)$ \\
\hline Hypoalbuminemia $(<3.5 \mathrm{~g} / \mathrm{dL})$ & $82 / 193(42.3)$ \\
\hline Renal failure (creatinine $>1.5 \mathrm{mg} / \mathrm{dL}$ ) & $18 / 193(9.3)$ \\
\hline \multicolumn{2}{|l|}{ Comorbidity (Charlson CSS) } \\
\hline $0-3$ & $146(75.3)$ \\
\hline$\geq 4$ & 48 (24.7) \\
\hline
\end{tabular}

Values are presented as number (\%) unless otherwise indicated. ECOG, Eastern Cooperative Oncology Group; LDH, lactate dehydrogenase; aaIPI, age-adjusted International Prognostic Index; CSS, comorbidity scoring system.

sex, presence of co-morbidities, B symptoms, bulky disease, Eastern Cooperative Oncology Group (ECOG) performance status, complete blood count, serum lactate dehydrogenase (LDH), creatinine, $\beta 2$-microglobulin, and albumin level, tumor stage, and site of extranodal disease. Staging was performed according to the criteria of the Ann Arbor system. Treatment pattern, response to treatment, toxicities, and outcome were obtained from the medical records of each patient. The Charlson comorbidity index was chosen to assess comorbidity [6].

A full dose of R-CHOP consisted of $375 \mathrm{mg} / \mathrm{m}^{2}$ rituximab, 
$750 \mathrm{mg} / \mathrm{m}^{2}$ cyclophosphamide, $50 \mathrm{mg} / \mathrm{m}^{2}$ adriamycin, and $1.4 \mathrm{mg} / \mathrm{m}^{2}$ vincristine on day 1 , and $100 \mathrm{mg}$ oral prednisone on days 1-5 of each cycle. The combination therapy was administered at 3-week intervals. The relative dose intensity (RDI) of these five drugs was calculated at each cycle. This study was approved by the institutional review board of each institution that participated in this study.

Overall survival was measured from the date of diagnosis of DLBCL to the date of death from any cause or the date of last follow-up. Survival curves were calculated using the Kaplan-Meier method and compared with other prognostic variables using the log-rank test. Stepwise Cox's regression analysis was performed to identify prognostic factors for survival. In all tests, $p<0.05$ was considered statistically significant.

\section{Results}

\section{Patient characteristics}

A total of 194 patients aged 80 years or older at diagnosis of DLBCL were identified. The patient characteristics are summarized in Table 1 . The study group included 93 men and 101 women, and the median age at the time of DLBCL diagnosis was 83.3 years (range, 80.1 to 95.7 years). One hundred and six patients (54.6\%) had a poor performance status (ECOG 2-4) and $58 \%$ of patients had advanced stage disease
Table 2. Treatment details

\begin{tabular}{|lc} 
Treatment & No. (\%) \\
\hline No treatment & $29(14.9)$ \\
\hline Operation (tumor removal) & $2(1.0)$ \\
Radiation alone & $9(4.6)$ \\
\hline First-line chemotherapy & 154 \\
R-CHOP & $124(63.9)$ \\
R-CVP & $13(6.7)$ \\
CVP & $8(4.1)$ \\
R alone & $1(0.5)$ \\
\hline Other & $8(4.1)$ \\
\hline Second-line chemotherapy & 26 \\
\hline Third-line chemotherapy & 4 \\
\hline
\end{tabular}

R-CHOP, rituximab, cyclophosphamide, adriamycin, vincristine, and prednisone; R, rituximab; CVP, cyclophosphamide, vincristine, and prednisone.

(stage III or IV). LDH level was elevated in $62 \%$ of patients, and $114(59.3 \%)$ patients had an age-adjusted International Prognostic Index (aaIPI) score of 2-3. Bone marrow biopsy was performed in 124 patients. Among these, eighteen patients showed bone marrow involvement $(14.5 \%)$. The left ventricular ejection fraction, measured in 123 patients at diagnosis, was below $60 \%$ in thirteen patients $(10.6 \%)$.

One hundred forty-nine patients $(76.8 \%)$ had at least one comorbidity. Hypertension was the most common comorbidity followed by diabetes mellitus and cerebrovascular dis-

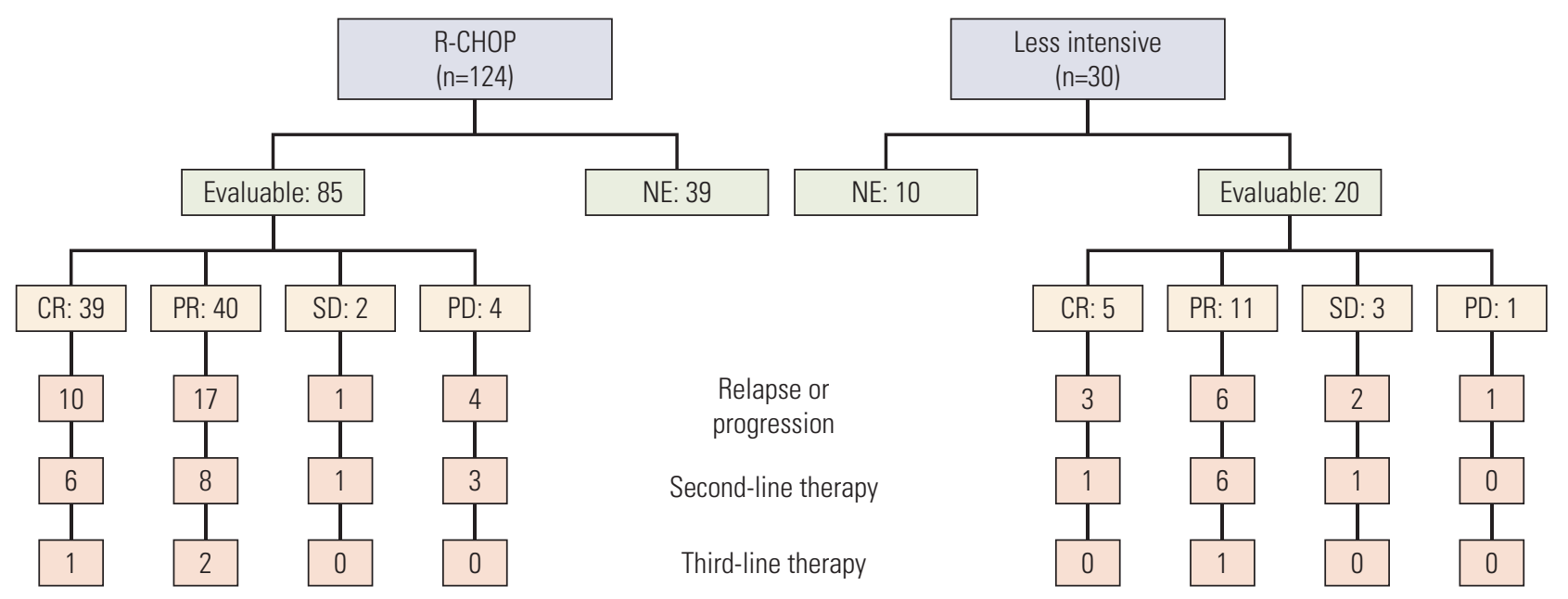

Fig. 1. Diagram of treatment and response for the patients who received chemotherapy. R-CHOP, rituximab, cyclophosphamide, adriamycin, vincristine, and prednisone; NE, not evaluable; $\mathrm{CR}$, complete response; $\mathrm{PR}$, partial response; $\mathrm{SD}$, stable disease; $\mathrm{PD}$, progressive disease. 
Table 3. Relative dose intensity and TRD of R-CHOP

\begin{tabular}{lccccccc} 
& \multirow{2}{*}{$\begin{array}{c}\text { No. of } \\
\text { patients }\end{array}$} & \multicolumn{5}{c}{ Relative dose intensity (\%) } \\
\cline { 3 - 7 } & & Rituximab & Cyclophosphamide & Adriamycin & Vincristine & Prednisone \\
First cycle & 124 & 86.8 & 68.2 & 64.1 & 74.1 & 84.0 \\
Second cycle & 95 & 84.8 & 65.0 & 60.0 & 70.4 & 80.1 & 2 \\
Third cycle & 76 & 83.2 & 64.5 & 58.0 & 68.9 & 80.5 & 3 \\
Fourth cycle & 53 & 81.3 & 62.5 & 58.0 & 68.3 & 80.7 & 0 \\
Fifth cycle & 35 & 85.4 & 60.8 & 56.7 & 64.7 & 77.9 & 4 \\
Sixth cycle & 24 & 96.9 & 69.6 & 65.5 & 80.3 & 89.9 \\
\hline
\end{tabular}

TRD, treatment-related death; R-CHOP, rituximab, cyclophosphamide, adriamycin, vincristine, and prednisone.

ease. Among the patients who presented with comorbidities, $48(24.7 \%)$ had a Charlson index with a score 4 or more.

\section{Treatments and outcomes}

Of 194 patients with DLBCL, 154 patients $(79.4 \%)$ received systemic chemotherapy, nine patients $(4.6 \%)$ received radiation alone, and two patients underwent surgery only (Table 2). Twenty-nine patients $(14.9 \%)$ received no treatment. The decision to forgo treatment was based on poor performance and/ or treatment refusal. Among 154 patients who received systemic chemotherapy, R-CHOP was given in 124 cases, R-CVP (rituximab, cyclophosphamide, vincristine, and prednisone) in 13, rituximab alone in one, and other regimens in 16 cases. Of 11 patients with central nervous system lymphoma, three cases were treated with high-dose metho-trexate with or without radiation.

The median number of chemotherapy cycles was three (range, 1 to 10). Of the 154 patients who received intravenous chemotherapy, only 37 completed the planned cycles of the treatment regimen. The main causes of treatment interruption were treatment-related toxicity $(45.3 \%)$ followed by treatment refusal $(29.1 \%)$ and disease progression $(6.8 \%)$. Forty-nine patients could not be evaluated for treatment response because of death before response assessment (18 cases), refusal (13 cases), follow-up loss (seven cases), and other causes (11 cases). The overall response rate for 154 patients who received systemic chemotherapy was $61.7 \%$. Among 105 patients who were available for response assessment, the response rate was $90.5 \%$ with a complete response (CR) rate of $41.9 \%$ (CR, 44; partial response [PR], 51; stable disease [SD], 5; progressive disease [PD], 5) (Fig. 1).

We performed subgroup analysis for 124 patients treated with R-CHOP because R-CHOP is standard treatment for DLBCL. The patients received a median of three cycles of R-CHOP (range, 1 to 8 ) and 13 patients with involved-field radiation therapy were combined. Thirty patients $(24.2 \%)$ completed the planned cycles and 24 patients $(19.4 \%)$ received six or more cycles of $\mathrm{R}-\mathrm{CHOP}$. The overall response rate for 124 patients who received R-CHOP was $63.7 \%$. Among 85 patients who were available for response assessment, the response rate was $92.9 \%$ with a CR rate of $45.9 \%$ (CR, 39; PR, 40; SD, 2; PD, 4).

We calculated the RDI of five drugs at each cycle of R-CHOP (Table 3). The mean RDIs for doxorubicin and cyclophosphiamide in the first cycle were $64.1 \%$ and $68.2 \%$, respectively. However, the RDIs of rituximab and prednisone were over $80 \%$. After the first cycle, the RDI of each drug showed a similar pattern to that of the first cycle. We compared the response rate and overall survival according to RDI for R-CHOP ( $\geq 70 \%$ vs. $<70 \%$ of all drugs). The overall response rate was significantly lower for high RDI group $(43.5 \%, \mathrm{n}=46)$ than for low RDI group $(75.6 \%, \mathrm{n}=78)(\mathrm{p}<0.001)$. The median survival was also significantly shorter for high RDI group than for low RDI group (11.1 months vs. 25.4 months, $p=0.008$ ). The reasons of these findings may be explained that high RDI group showed higher treatment interruption rate and treatment-related mortality. Especially, $52.2 \%$ of high RDI group received only one cycle of R-CHOP.

Among the 44 patients who relapsed or progressed after first-line therapy, 26 patients received salvage therapy. Twenty-two patients were treated with various chemotherapies and three patients were treated with radiotherapy. One patient received combined treatment. The overall response rate for the salvage therapy was $61.1 \%$. Four patients received third-line treatment (Fig. 1).

\section{Toxicity profiles}

The most common side effect of chemotherapy was hematologic toxicity. Neutropenia was reported in 63.0\% (97/154) of patients, $91.8 \%$ (89/97) of whom had grade 3 or higher toxicity. Granulocyte colony-stimulating factor (G-CSF) was given to 73 patients during the first cycle. Forty-eight patients 
A

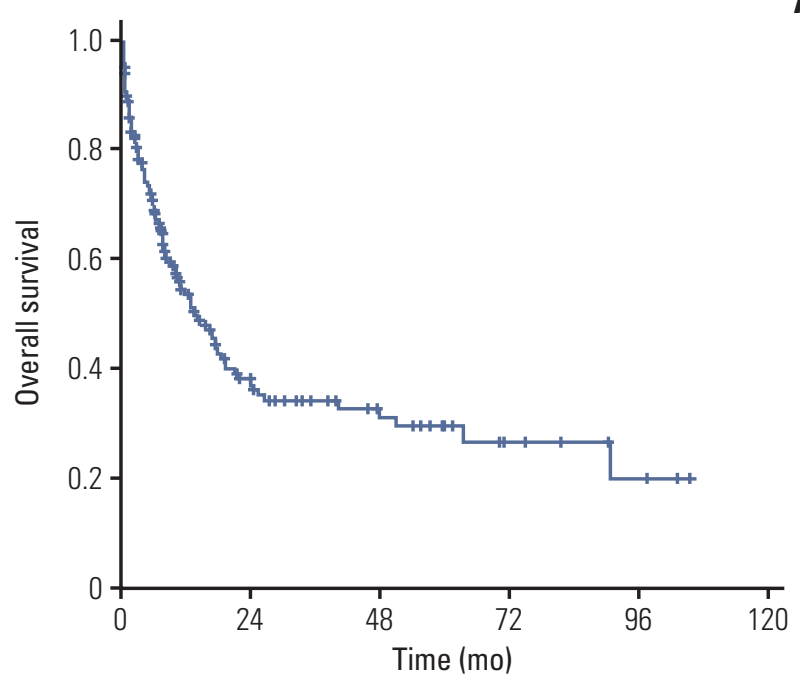

C

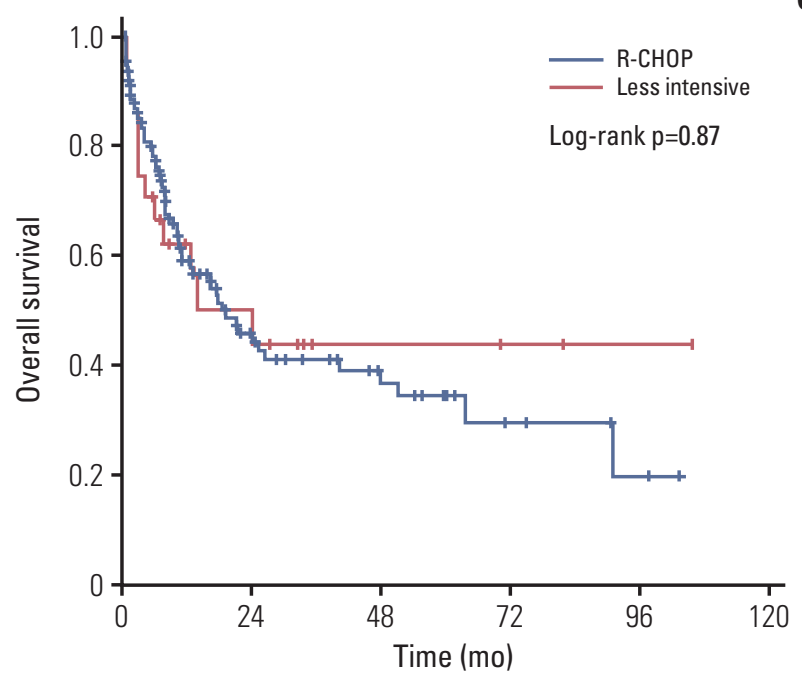

B

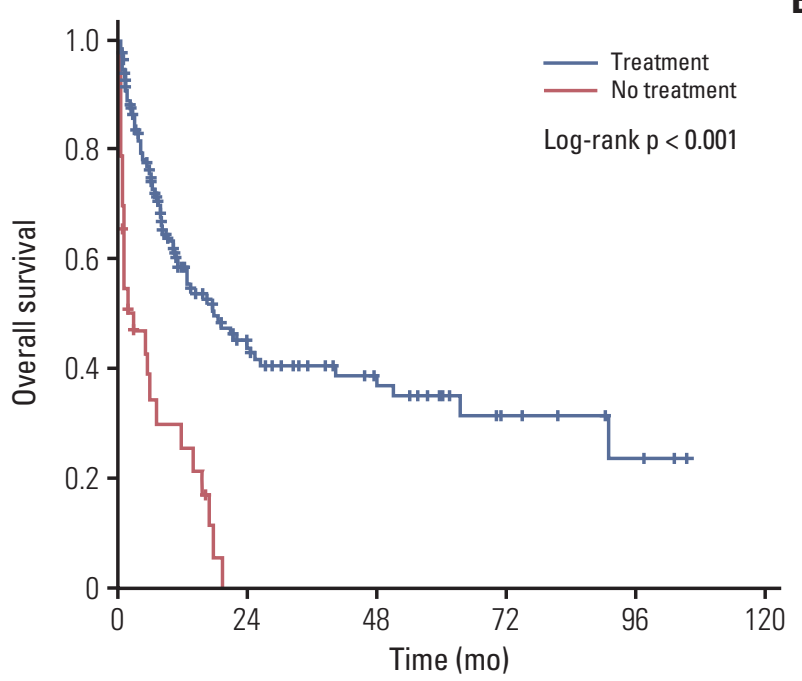

D

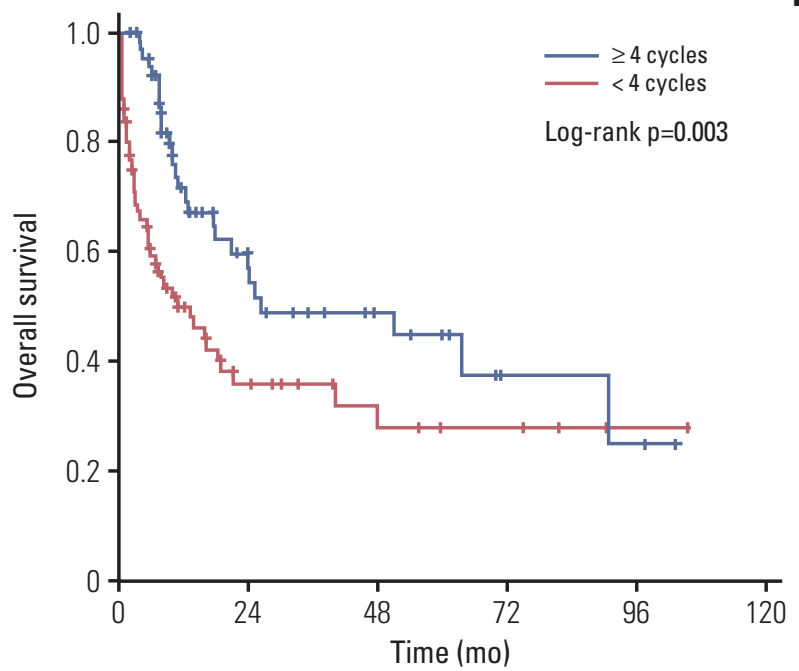

Fig. 2. Kaplan-Meier plots of overall survival in 194 patients (A) and according to treatment (B). Overall survival in 154 patients who received chemotherapy according to treatment regimen (C) and number of chemotherapy cycles (D).

$(31.2 \%)$ suffered neutropenic fever. Anemia was reported in $59.7 \%$ (92/154) and thrombocytopenia in $33.1 \%$ (51/154). The incidence of grade 3 or 4 toxicity of anemia and thrombocytopenia was relatively lower than that of neutropenia ( $27.2 \%$ and $35.3 \%$, respectively). Infection was documented in 47 cases. Pneumonia (55.3\%) was the most common infectious complication. Cardiac toxicity occurred in five cases.

Twenty-nine patients died due to treatment-related toxicity. Among them, 13 patients died after the first cycle of chemotherapy and the main causes of death were pneumonia and sepsis (10 cases). Of 124 patients treated with $\mathrm{R}-\mathrm{CHOP}$, the treatment-related mortality rate was $16.9 \%$
$(21 / 124)$. However, among 19 patients who received the full dose of R-CHOP, six patients died due to toxicity (31.6\%).

\section{Survival analyses}

One hundred and seven patients died with a median survival of 14.0 months (Fig. 2). Of 124 patients treated with $\mathrm{R}-\mathrm{CHOP}$, median overall survival was 18.5 months. The main causes of death were disease progression (33 cases, $30.8 \%$ ) and treatment-related toxicity (29 cases, $27.1 \%$ ). The cause of death according to treatment is shown in Table 4. In the univariate analysis, significant prognostic factors for 
Table 4. Cause of death according to treatment

\begin{tabular}{|c|c|c|c|c|c|c|}
\hline \multirow{2}{*}{ Treatment } & \multirow{2}{*}{$\begin{array}{c}\text { No. of } \\
\text { patients }\end{array}$} & \multirow{2}{*}{ Death } & \multirow{2}{*}{$\begin{array}{c}\text { Disease } \\
\text { progression }\end{array}$} & \multicolumn{3}{|c|}{ Cause of death } \\
\hline & & & & Toxicity & Other cause & Unknown \\
\hline R-CHOP & 124 & 63 & 17 & $22^{\text {a) }}$ & 10 & 14 \\
\hline Other treatment & 41 & 19 & 3 & 7 & 3 & 6 \\
\hline No treatment & 29 & 25 & 13 & 0 & 6 & 6 \\
\hline Total & 194 & 107 & 33 & 29 & 19 & 26 \\
\hline
\end{tabular}

R-CHOP, rituximab, cyclophosphamide, adriamycin, vincristine, and prednisone. ${ }^{\mathrm{a})}$ One patient died due to treatment-related toxicity after salvage therapy.

Table 5. Univariate analysis of prognostic factors for overall survival

\begin{tabular}{|c|c|c|}
\hline Prognostic factor & Survival (median \pm SE, mo) & p-value \\
\hline \multicolumn{3}{|l|}{ aaIPI } \\
\hline 0 & Not reached & $<0.001$ \\
\hline 1 & $19.3 \pm 5.1$ & \\
\hline 2 & $14.0 \pm 6.5$ & \\
\hline 3 & $7.7 \pm 2.8$ & \\
\hline \multicolumn{3}{|l|}{ ECOG } \\
\hline $0-1$ & $24.3 \pm 11.2$ & $<0.001$ \\
\hline $2-4$ & $7.9 \pm 1.7$ & \\
\hline \multicolumn{3}{|l|}{ Stage } \\
\hline I-II & $18.5 \pm 6.5$ & 0.088 \\
\hline III-IV & $11.1 \pm 2.2$ & \\
\hline \multicolumn{3}{|l|}{ LDH } \\
\hline Normal & $18.5 \pm 3.0$ & 0.016 \\
\hline Elevated & $10.7 \pm 2.4$ & \\
\hline \multicolumn{3}{|c|}{ No. of extranodal sites } \\
\hline$<2$ & $16.0 \pm 2.7$ & 0.032 \\
\hline$\geq 2$ & $9.3 \pm 1.8$ & \\
\hline \multicolumn{3}{|l|}{ Hemoglobin (g/dL) } \\
\hline$<12$ & $11.1 \pm 1.9$ & 0.005 \\
\hline$\geq 12$ & 21.6 & \\
\hline \multicolumn{3}{|l|}{ Albumin (g/dL) } \\
\hline$<3.5$ & $6.4 \pm 0.9$ & $<0.001$ \\
\hline$\geq 3.5$ & $21.0 \pm 3.3$ & \\
\hline \multicolumn{3}{|l|}{ Creatinine (mg/dL) } \\
\hline$<1.5$ & $16.9 \pm 2.6$ & 0.001 \\
\hline$\geq 1.5$ & $4.6 \pm 2.8$ & \\
\hline \multicolumn{3}{|c|}{ Comorbidity (Charlson CSS) } \\
\hline$<4$ & $17.5 \pm 3.1$ & 0.013 \\
\hline$\geq 4$ & $10.4 \pm 1.8$ & \\
\hline \multicolumn{3}{|l|}{ Treatment } \\
\hline R-CHOP & $18.5 \pm 3.8$ & $<0.001$ \\
\hline Other treatment & $12.9 \pm 4.3$ & \\
\hline No treatment & $2.7 \pm 1.9$ & \\
\hline
\end{tabular}

SE, standard error; aaIPI, age-adjusted International Prognostic Index; ECOG, Eastern Cooperative Oncology Group; LDH, lactate dehydrogenase; CSS, comorbidity scoring system; R-CHOP, rituximab, cyclophosphamide, adriamycin, vincristine, and prednisone. 
Table 6. Multivariate analysis of prognostic factors for overall survival

\begin{tabular}{lcr} 
Prognostic factor & Hazard ratio (95\% CI) & p-value \\
\hline aaIPI & $1.358(1.082-1.704)$ & 0.008 \\
Anemia & $1.276(0.812-2.005)$ & 0.291 \\
Hypoalbuminemia & $1.991(1.292-3.069)$ & 0.002 \\
Increased creatinine & $2.568(1.424-4.632)$ & 0.002 \\
Comorbidity (Charlson CSS) & $1.540(0.987-2.403)$ & 0.057 \\
\hline No treatment & $1.642(1.274-2.117)$ & $<0.001$ \\
\hline
\end{tabular}

CI, confidence interval; aaIPI, age-adjusted International Prognostic Index; CSS, comorbidity scoring system.

overall survival were ECOG performance status, serum LDH, number of extranodal sites, aaIPI, anemia, hypoalbuminemia, elevated creatinine, co-morbidity, and treatment (Table 5). There was no significant survival difference between $\mathrm{R}-\mathrm{CHOP}$ and less intensive treatment group $(\mathrm{p}=0.870)$ (Fig. 2C). However, we found significant difference in the median survival according to number of chemotherapy cycles ( $<4$ cycles vs. $\geq 4$ cycles) (11.1 months vs. 26.6 months, $\mathrm{p}=0.003$ ) (Fig. 2D). In multivariate analyses using the significant prognostic variables from univariate analysis, overall survival was affected by aaIPI, hypoalbuminemia, elevated creatinine, and treatment (Table 6).

\section{Discussion}

In this study, we found that more than half of very elderly patients with DLBCL (63.9\%) who received R-CHOP had a high response rate of $92.9 \%$ (CR, 45.9\%) in Korea. However, our study showed a high treatment-related mortality rate due to infection with severe neutropenia, especially after the first cycle of chemotherapy. Significant prognostic factors for overall survival included aaIPI, hypoalbuminemia, elevated creatinine, and treatment.

The treatment strategy for elderly patients older than 80 years with DLBCL has not been defined yet because of poor treatment tolerability and lack of data. Retrospective studies have reported that elderly patients have worse outcomes due to suboptimal therapy and toxicity; however, some patients achieved a CR and long-term survival [7,8]. There have been several trials that sought to reduce toxicity and to identify optimal regimens in elderly patients. "Prephase treatment" has been proposed in order to reduce "first-cycle effects," which refer to side effects of the first chemotherapy cycle such as the deepest neutrophil nadir, longest neutropenic duration, tumor lysis syndrome and highest rate of treatment-related mortality [9]. Prephase treatment consisted of oral prednisone for 5 to 7 days alone or with single injection of $1 \mathrm{mg}$ vincristine before the first chemotherapy cycle. After introduction of the prephase treatment, the number of treatment-related deaths was significantly decreased and the performance status was improved. Therefore, the author recommends prephase treatment for all elderly patients [10].

In the search for adequate therapy of very elderly or frail patients, several alternative regimens have been reported [11-15]. Among them, R-miniCHOP data showed good results of both efficacy and safety in 149 elderly patients older than 80 years with DLBCL. This study reported that the median overall survival was 29 months with a $62 \%$ CR and unconfirmed $\mathrm{CR}$ rate. However, only 12 deaths were related to treatment toxicity [3]. R-miniCHOP consisted of $375 \mathrm{mg} / \mathrm{m}^{2}$ rituximab, $400 \mathrm{mg} / \mathrm{m}^{2}$ cyclophosphamide, $25 \mathrm{mg} / \mathrm{m}^{2}$ adriamycin, and $1 \mathrm{mg}$ vincristine on day 1 , and $40 \mathrm{mg} / \mathrm{m}^{2}$ oral prednisone on days 1 through 5 of each cycle at 3-week intervals. The doses of each drug were similar to the mean dose intensity of R-CHOP in our study. In addition, our data revealed high treatment-related mortality in patients treated with full dose R-CHOP. Therefore, we suggest that R-miniCHOP is an acceptable regimen for very elderly patients based on results from actual clinical practice.

In addition, supportive care including careful monitoring, prophylaxis and treatment for infection should be included to reduce morbidity and mortality. The American Society of Clinical Oncology guideline for the use of G-CSF recommends prophylactic G-CSF in elderly patients receiving CHOP or R-CHOP [16]. In our study, grade 3 or 4 neutropenia was the most common severe side effect of chemotherapy and infection-associated neutropenia was the most common cause of treatment-related death. More than three-fourths of neutropenia cases $(78.4 \%)$ developed after the first cycle and 13 patients died due to toxicity just after the first cycle. These results demonstrate that prophylactic G-CSF is essential for treating $\mathrm{R}-\mathrm{CHOP}$ in elderly patients.

The therapeutic goal in treating very elderly DLBCL patients is to balance between efficacy and toxicity of treatment. Therefore, it is important to identify patients who will 
tolerate and respond to treatment. A low serum albumin level is a significant parameter associated with poor outcome in our study. The finding that hypoalbuminemia is a poor prognostic factor was consistent with results from previous reports $[3,4]$. Hypoalbuminemia reflects malnutrition, inflammation and advanced disease. Therefore, a low serum albumin concentration might be an important parameter for the ability to tolerate treatment.

In the present study, the CR rate was lower than that from a previous study. The cause of the lower $\mathrm{CR}$ rate may be high incidence of treatment interruption and reduced dose intensity. Response to first-line therapy is more important in elderly patients since adequate salvage therapy is usually difficult in these patients. Therefore, a well-designed prospective randomized trials for searching optimal regimens for the very elderly patients with DLBCL is needed.

The present study had several limitations. First, the clinical data, including treatment, side effects, relapse and survival data, were analyzed retrospectively. There were concerns of under-reporting and reporting bias, especially with toxicity profiles and patients lost to follow-up. In addition, treatment modality was not pre-determined. However, this study is one of the largest analyses of DLBCL patients older than 80 years and our results reflect the actual clinical practice in Korea.

These results suggest that age itself should not be a major influence on treatment decision-making by clinicians and adequate therapy should be considered in elderly DLBCL patients. However, since elderly patients show higher treatment-related mortality due to infection, careful monitoring and dose modification of chemotherapeutic agents is needed. Based on these results, we plan to perform a prospective study of R-miniCHOP with prephase treatment and prophylactic G-CSF in very elderly patients older than 80 years with DLBCL in Korea.

\section{Conflicts of Interest}

Conflict of interest relevant to this article was not reported.

\section{Acknowledgments}

This research was supported in part by the Korean Cancer Study Group.

\section{Author Details}

${ }^{1}$ Department of Internal Medicine, Hanyang University Guri Hospital, Hanyang University College of Medicine, Guri, ${ }^{2}$ Department of Internal Medicine, Seoul National University Hospital, Seoul, ${ }^{3}$ Department of Internal Medicine, Hallym University Sacred Heart Hospital, Hallym University College of Medicine, Anyang, ${ }^{4}$ Department of Internal Medicine, Yeungnam University Medical Center, Daegu, ${ }^{5}$ Department of Internal Medicine, Ewha Womans University College of Medicine, Seoul, ${ }^{6}$ Department of Internal Medicine, Korea Institute of Radiological and Medical Sciences, Korea Cancer Center Hospital, Seoul, ${ }^{7}$ Department of Internal Medicine, Yeouido St. Mary's Hospital, College of Medicine, The Catholic University of Korea, Seoul, ${ }^{8}$ Department of Internal Medicine, Wonkwang University School of Medicine, Iksan, ${ }^{9}$ Department of Internal Medicine, CHA Bundang Medical Center, CHA University, Seongnam, ${ }^{10}$ Department of Internal Medicine, Uijeongbu St. Mary's Hospital, College of Medicine, The Catholic University of Korea, Uijeongbu, ${ }^{11}$ Department of Internal Medicine, Dankook University Hospital, Dankook University College of Medicine, Cheonan, ${ }^{12}$ Department of Internal Medicine, Hanyang University College of Medicine, Seoul, ${ }^{13}$ Department of Internal Medicine, Hallym University Kangdong Sacred Heart Hospital, Hallym University College of Medicine, Seoul, ${ }^{14}$ Department of Internal Medicine, Veterans Health Service Medical Center, Seoul, ${ }^{15}$ Department of Internal Medicine, Chung-Ang University Hospital, Seoul, ${ }^{16}$ Department of Internal Medicine, Inje University Sanggye Paik Hospital, Seoul, ${ }^{17}$ Department of Internal Medicine, Bucheon St. Mary's Hospital, College of Medicine, The Catholic University of Korea, Bucheon, ${ }^{18}$ Devision of Hematology-Oncology, Department of Internal Medicine, Kyung Hee University School of Medicine, Seoul, ${ }^{19}$ Department of Internal Medicine, Samsung Changwon Hospital, Sungkyunkwan University School of Medicine, Changwon, Korea

\section{References}

1. Coiffier B, Lepage E, Briere J, Herbrecht R, Tilly H, Bouabdallah $\mathrm{R}$, et al. CHOP chemotherapy plus rituximab compared with CHOP alone in elderly patients with diffuse large-B-cell lymphoma. N Engl J Med. 2002;346:235-42.

2. Feugier P, Van Hoof A, Sebban C, Solal-Celigny P, Bouabdallah R, Ferme C, et al. Long-term results of the R-CHOP study in the treatment of elderly patients with diffuse large B-cell lymphoma: a study by the Groupe d'Etude des Lymphomes de l'Adulte. J Clin Oncol. 2005;23:4117-26.

3. Peyrade F, Jardin F, Thieblemont C, Thyss A, Emile JF, Castaigne $S$, et al. Attenuated immunochemotherapy regimen (R-miniCHOP) in elderly patients older than 80 years with diffuse large B-cell lymphoma: a multicentre, single-arm, phase 2 trial. Lancet Oncol. 2011;12:460-8. 
4. Jung YH, Woo IS, Han CW. Clinical characteristics and outcomes in diffuse large B cell lymphoma patients aged 70 years and older: a single-center experience with a literature review. Korean J Intern Med. 2015;30:684-93.

5. Ha H, Keam B, Kim TM, Jeon YK, Lee SH, Kim DW, et al. Reduced dose intensities of doxorubicin in elderly patients with DLBCL in rituximab era. Cancer Res Treat. 2016;48: 304-11.

6. Charlson ME, Pompei P, Ales KL, MacKenzie CR. A new method of classifying prognostic comorbidity in longitudinal studies: development and validation. J Chronic Dis. 1987;40: 373-83.

7. Bairey O, Benjamini O, Blickstein D, Elis A, Ruchlemer R. NonHodgkin's lymphoma in patients 80 years of age or older. Ann Oncol. 2006;17:928-34.

8. Thieblemont C, Grossoeuvre A, Houot R, Broussais-Guillaumont F, Salles G, Traulle C, et al. Non-Hodgkin's lymphoma in very elderly patients over 80 years: a descriptive analysis of clinical presentation and outcome. Ann Oncol. 2008;19:774-9.

9. Pfreundschuh M, Trumper L, Kloess M, Schmits R, Feller AC, Rube C, et al. Two-weekly or 3-weekly CHOP chemotherapy with or without etoposide for the treatment of elderly patients with aggressive lymphomas: results of the NHL-B2 trial of the DSHNHL. Blood. 2004;104:634-41.

10. Pfreundschuh M. How I treat elderly patients with diffuse large B-cell lymphoma. Blood. 2010;116:5103-10.

11. Morrison VA, Hamlin P, Soubeyran P, Stauder R, Wadhwa P, Aapro M, et al. Approach to therapy of diffuse large B-cell lymphoma in the elderly: the International Society of Geriatric Oncology (SIOG) expert position commentary. Ann Oncol.
2015;26:1058-68.

12. Hainsworth JD, Flinn IW, Spigel DR, Clark BL, Griner PL, Vazquez ER, et al. Brief-duration rituximab/chemotherapy followed by maintenance rituximab in patients with diffuse large B-cell lymphoma who are poor candidates for R-CHOP chemotherapy: a phase II trial of the Sarah Cannon Oncology Research Consortium. Clin Lymphoma Myeloma Leuk. 2010;10:44-50.

13. Musolino A, Boggiani D, Panebianco M, Vasini G, Salvagni S, Franciosi $\mathrm{V}$, et al. Activity and safety of dose-adjusted infusional cyclophosphamide, doxorubicin, vincristine, and prednisone chemotherapy with rituximab in very elderly patients with poor-prognostic untreated diffuse large B-cell nonHodgkin lymphoma. Cancer. 2011;117:964-73.

14. Weidmann E, Neumann A, Fauth F, Atmaca A, Al-Batran SE, Pauligk $C$, et al. Phase II study of bendamustine in combination with rituximab as first-line treatment in patients 80 years or older with aggressive B-cell lymphomas. Ann Oncol. 2011;22:1839-44.

15. Fields PA, Townsend W, Webb A, Counsell N, Pocock C, Smith $\mathrm{P}$, et al. De novo treatment of diffuse large B-cell lymphoma with rituximab, cyclophosphamide, vincristine, gemcitabine, and prednisolone in patients with cardiac comorbidity: a United Kingdom National Cancer Research Institute trial. J Clin Oncol. 2014;32:282-7.

16. Smith TJ, Bohlke K, Lyman GH, Carson KR, Crawford J, Cross SJ, et al. Recommendations for the use of WBC growth factors: American Society of Clinical Oncology clinical practice guideline update. J Clin Oncol. 2015;33:3199-212. 